\title{
NONCOINCIDENCE OF THE STRICT AND STRONG OPERATOR TOPOLOGIES
}

\author{
JOEL H. SHAPIRO
}

\begin{abstract}
Let $E$ be an infinite-dimensional linear subspace of $C(S)$, the space of bounded continuous functions on a locally compact Hausdorff space $S$. If $\mu$ is a regular Borel measurc on $S$, then each element of $E$ may be regarded as a multiplication operator on $L^{p}(\mu)(1 \leqq p<\infty)$. Our main result is that the strong operator topology this identification induces on $E$ is properly weaker than the strict topology. For $E$ the space of bounded analytic functions on a plane region $G$, and $\mu$ Lebesgue measure on $G$, this answers negatively a question raised by Rubel and Shields in [9]. In addition, our methods provide information about the absolutely $p$-summing properties of the strict topology on subspaces of $C(S)$, and the bounded weak star topology on conjugate Banach spaces.
\end{abstract}

1. Introduction. Let $C(S)$ denote the space of bounded, continuous, complex valued functions on a locally compact Hausdorff space $S$, and let $C_{0}(S)$ denote those functions in $C(S)$ which vanish at infinity. The strict topology $\beta$ on $C(S)$ is the locally convex topology induced by the seminorms

$$
f \rightarrow\|f k\|_{\infty} \quad(f \in C(S)),
$$

where $k$ runs through $C_{0}(S)$ and $\|\cdot\|_{\infty}$ denotes the supremum norm. This topology was introduced in [1] by Buck who derived many of its funda, mental properties. In particular [1, Theorems 1 and 2]: $\beta$ is completeHausdorff, and weaker than the norm topology; the norm and strictly bounded subsets of $C(S)$ coincide, and the $\beta$-dual of $C(S)$ can be identified with $M(S)$, the space of finite, regular Borel measures on $S$, where the pairing between the spaces is

$$
f, \mu\rangle=\int f d \mu \quad(f \in C(S), \mu \in M(S)) .
$$

Let $\mu$ be a (possibly infinite) regular Borel measure on $S$, as defined in [4, Section 52]. Note that built into this definition is the fact that $\mu(K)<\infty$

Received by the editors February 9, 1972.

AMS 1969 subject classifications. Primary 4601, 4625; Secondary 46.30

Key words and phrases. Strict topology, strong operator topolog!, absolutely $p$ summing topology, bounded continuous functions. 
for every compact subset $K$ of $S$ [4, p. 223]. For each $f$ in $C(S)$ the equation $M_{f} g=f g\left(g \in L^{p}(\mu)\right)$ defines a bounded linear operator $M_{f}$ on $L^{p}(\mu)$. It is not difficult to see from the fact that $\mu$ gives finite measure to compact sets that the linear map $f \rightarrow M_{f}$ is actually an isometry taking $C(S)$ into the space of all bounded linear operators on $L^{p}(\mu)$. Thus $C(S)$ inherits the strong operator topology $\sigma_{p}=\sigma_{p}(\mu)$, defined by the seminorms

$$
f \rightarrow\left\{\int|f g|^{p} d \mu\right\}^{1 / p} \quad(f \in C(S))
$$

where $g$ runs through $L^{p}(\mu)\left[3\right.$, VI. 1.2, p. 475]. Note that $\sigma_{p}$ is locally convex and Hausdorff.

Now $C(S)$ also acts on $C_{0}(S)$ by multiplication, and in this case the corresponding strong operator topology is the strict topology. In [9, 5.18(c), p. 274], Rubel and Shields asked if $\beta=\sigma_{2}(\mu)$ on the space $H^{\infty}(G)$, where $\mu$ is two-dimensional Lebesgue measure on $G$, and $G$ supports nonconstant bounded analytic functions. In this case $H^{\infty}(G)$ is infinite dimensional [9, Section 2.3], so the question is answered in the negative by the following theorem, which is our main result.

THEOREM 1. Let $E$ be an infinite-dimensional linear subspace of $C(S)$, and suppose $\mu$ is a regular Borel measure on $S$. Then the strong operator topology $\sigma_{p}(\mu)$ induced on $E$ by its action on $L^{p}(\mu)$ is properly weaker than the strict topology.

The proof of this result occupies $\S 3$, and uses the notion of absolutely $p$-summing locally convex topologies, introduced in the next section. In $\S 4$ we comment briefly on the bounded strong operator topology and the bounded weak star topology.

2. Absolutely $p$-summing topologies. Let $\tau$ be a locally convex topology on a real or complex linear space $E$, and let $E^{\prime}=E_{\tau}^{\prime}$ denote the $\tau$-dual of $E$ (all $\tau$-continuous linear functionals on $E$ ). For $e^{\prime}$ in $E^{\prime}$ and $e$ in $E$ we will write $\left\langle e, e^{\prime}\right\rangle$ instead of $e^{\prime}(e)$. A sequence $\left(e_{n}\right)$ in $E$ is called $\tau$-weakly $p$ summable if $\sum\left|\left\langle e_{n}, e^{\prime}\right\rangle\right|^{p}<\infty$ for all $e^{\prime}$ in $E^{\prime}$, and $\tau$-absolutely $p$-summable if $\sum S\left(e_{n}\right)^{p}<\infty$ for every $\tau$-continuous seminorm $S$ on $E(1 \leqq p<\infty)$. If every $\tau$-weakly $p$-summable sequence is $\tau$-absolutely $p$-summable, we say $\tau$ is absolutely p-summing. For example, the weak topology on a Banach space is absolutely $\rho$-summing for all $p$; but if the space is infinitedimensional, then the Dvoretzky-Rogers theorem [8, Theorem 8, p. 350] asserts that the norm topology is absolutely $p$-summing for no $p(1 \leqq p<\infty)$. Note that if $\tau$ is not absolutely $p$-summing, then neither is any stronger locally convex topology on $E$ with the same continuous linear functionals. 
The following lemma, which is an easy consequence of the DvoretzkyRogers theorem, is the key to our proof of Theorem 1. We note that the same idea has been used in [6, Example 2, p. 417].

LEMMA 1. Let $E$ be an infinite-dimensional normed space, and let $F$ be $a$ linear subspace of $E^{\prime}$ which norms $E$; that is,

$$
\|e\|=\sup \{|\langle e, f\rangle|: f \in F,\|f\| \leqq 1\}
$$

for each $e$ in $E$. Let $\tau$ denote the topology on $E$ of uniform convergence on (norm) null sequences of $F$. Then $\tau$ is not absolutely p-summing $(1 \leqq p<\infty)$.

Proof. Since $E$ is infinite-dimensional it follows from the DvoretzkyRogers theorem stated above that there is a sequence $\left(e_{n}\right)$ in $E$ which is weakly, but not absolutely, $p$-summable for the norm topology; that is, $\sum\left|\left\langle e_{n}, e^{\prime}\right\rangle\right|^{p}<\infty$ for all $e^{\prime}$ in $E^{\prime}$, but $\sum\left\|e_{n}\right\|^{p}=\infty$. Since $\tau$ is weaker then the norm topology, every $\tau$-continuous linear functional on $E$ is norm continuous; hence $\left(e_{n}\right)$ is $\tau$-weakly $p$-summable. We claim that $\left(e_{n}\right)$ is not $\tau$-absolutely $p$-summable. For by $(2.1)$ there exists $f_{n}$ in $F$ with $\left\|f_{n}\right\| \leqq 1$, and

$$
\left|\left\langle e_{n}, f_{n}\right\rangle\right|>\left\|e_{n}\right\| / 2^{1 / p} \quad(n=1,2, \cdots) .
$$

Let $\left(a_{n}\right)$ be a sequence of nonnegative numbers such that $\lim a_{n}=0$, and $\sum a_{n}^{p}\left\|e_{n}\right\|^{p}=\infty$, and let $g_{n}=a_{n} f_{n}(n=1,2, \cdots)$. Then lim $\left\|g_{n}\right\|=0$, so the equation $S e=\sup _{n}\left|\left\langle e, g_{n}\right\rangle\right|(e$ in $E)$ defines a $\tau$-continuous seminorm on $E$. But

$$
\begin{aligned}
\sum\left(S e_{n}\right)^{p} & \geqq \sum\left|\left\langle e_{n}, g_{n}\right\rangle\right|^{p}=\sum a_{n}^{p}\left|\left\langle e_{n}, f_{n}\right\rangle^{p}\right|^{p} \\
& \geqq \sum a_{n}^{p}\left\|e_{n}\right\|^{p} / 2=\infty,
\end{aligned}
$$

so $\tau$ is not absolutely $p$-summing.

We will also need a result of J. B. Conway concerning factorization of subsets of $M(S)$. Recall that a subset $H$ of $M(S)$ is called tight if for each $\varepsilon>0$ there exists a compact subset $K$ of $S$ such that $|\mu|(S-K)<\varepsilon$ for each $\mu$ in $H$.

Lemma 2 [2, Throrem 2.2, P. 476]. A bounded subset $H$ of $M(S)$ is tight if and only if there is a bounded subset $B$ of $M(S)$ and a function $k$ in $C_{0}(S)$ such that $H=k B$.

Here, of course, $k B=\{k b: b \in B\}$. We can now prove the main result of this section.

Proposition 1. Let E be an infinite-dimensional linear subspace of $C(S)$. Then the strict topology on $E$ is not absolutely p-summing $(1 \leqq p<\infty)$. 
Proof. Since the strict dual of $C(S)$ is $M(S)$, where the spaces are paired by (1.1) [1, Theorem 2], it follows easily that the strict dual $E_{\beta}^{\prime}$ of $E$ may be identified with the quotient space $M(S) / E^{\circ}$, via the pairing

$$
\left\langle\int, \alpha+E^{\circ}\right\rangle=\int f d \alpha \quad(f \in E, \alpha \in M(S)),
$$

where $E^{\circ}$ is the annihilator of $E$ in $M(S)$ (see [5, Theorem 14.5, p. 120]). Moreover $E_{\beta}^{\prime}$ is a subspace of $E^{\prime}$, the norm dual of $E$, so it is a normed space.

We will need the fact that for each $\alpha$ in $M(S)$ the norm of the coset $\alpha+E^{\circ}$ viewed as a linear functional on $E$ coincides with its norm as an element of $M(S) / E^{\circ}$. To see this, note that each $e$ in $E$ acts by integration as a linear functional on $M(S)$ of norm $\|e\|$, so the pairing (1.1) induces an isometric isomorphism of $E$ into $M(S)^{\prime}$. Standard Banach space theory now shows that the weak star closure $\bar{E}$ of $E$ in $M(S)^{\prime}$ is isometrically isomorphic to the dual of $M(S) / \bar{E}^{\circ}$, where $\bar{E}^{\circ}$ is the annihilator of $\bar{E}$ in $M(S)$. But $\bar{E}^{\circ}=E^{\circ}$, which proves our assertion.

Now the evaluation functionals $\left(\lambda_{s}: s \in S\right)$ defined by

$$
\lambda_{s}(e)=e(s) \quad(e \text { in } E)
$$

are strictly continuous and have norm $\leqq 1$, so $E_{\beta}^{\prime}$ norms $E$ in the sense of Lemma 1; hence Lemma 1 shows that the topology $\tau$ of uniform convergence on norm null sequences in $E_{\beta}^{\prime}$ is not absolutely $p$-summing. Clearly $\tau$ is stronger than the weak topology induced on $E$ by $E_{\beta}^{\prime}$, so we will be finished if we prove that $\tau \leqq \beta$; for then $E_{\tau}^{\prime}=E_{\beta}^{\prime}$, hence $\beta$ is not absolutely $p$-summing since $\tau$ is not.

To show that $\tau \leqq \beta$, suppose $\left(e_{n}^{\prime}\right)$ is a norm null sequence in $E_{\beta}^{\prime}$. By the isometric identification of $E_{\beta}^{\prime}$ with $M(S) / E^{\circ}$ there exists a sequence $\left(\alpha_{n}\right)$ in $M(S)$ such that $\lim \left\|\alpha_{n}\right\|=0$, and for each $n,\left\langle e, e_{n}^{\prime}\right\rangle=\int e d \alpha_{n}(e$ in $E)$. It is easy to see that (the range of) $\left(\alpha_{n}\right)$ is tight, hence by Lemma 2 there is a bounded sequence $\left(\lambda_{n}\right)$ in $M(S)$ and a function $k$ in $C_{0}(S)$ such that $\alpha_{n}=$ $k \lambda_{n}$ for all $n$. Thus for $e$ in $E$,

$$
\sup _{n}\left|\vdots e, e_{n}^{\prime}\right\rangle=\sup _{n}\left|\int e k d \lambda_{n}\right| \leqq\|e k\|_{\infty} \sup _{n}\left\|\lambda_{n}\right\| .
$$

Since the left side of this inequality is a typical $\tau$-seminorm, and the right side is a $\beta$-continuous seminorm, we have $\tau \leqq \beta$.

Note that Proposition 1 shows in particular that on any infinitedimensional linear subspace $E$ of $C(S)$ the strict topology is not nuclear. This fact was first conjectured by Klaus D. Bierstedt for $E=H^{\infty}(D), D$ the open unit disc (private communication). 
3. Proof of Theorem 1. For convenience we replace the function $g$ in (1.2) by $|g|^{p}$. Thus the topology $\sigma_{n}=\sigma_{p}(\mu)$ is induced by the seminorms

$$
S_{g}(f)=\left\{\int|f|^{p} g d \mu\right\}^{1 / p} \quad(f \in E),
$$

where $g$ runs through $L^{+}$, the class of nonnegative $\mu$-integrable functions on $S$. Since the maximum of two $L^{+}$functions is again in $L^{+}$, we see easily that the sets

$$
\left\{f \in C(S): S_{g} f \leqq 1\right\} \quad\left(g \in L^{\dagger}\right)
$$

form a base for the $\sigma_{p}$-neighborhoods of zero in $E$.

Now if $g \in L^{\dagger}$, then it follows from the regularity of $\mu$ that $g \mu \in M(S)$. By the argument used in the proof of Lemma 2 [2, Theorem 2.2] with $H=$ $\{g \mu\}$, there exists $k$ in $C_{0}(S)$ and $h$ in $L^{+}$such that $g=k^{p} h$. Thus

$$
S_{g} f \leqq\|f k\|_{\infty}\|h\|_{1}^{1 / p} \quad(f \in C(S)),
$$

so $\sigma_{p} \leqq \beta$ on $C(S)$.

We complete the proof by showing that $\sigma_{p} \neq \beta$ on $E$ whenever $E$ is infinite-dimensional. If the strict dual $E_{\beta}^{\prime}$ of $E$ is different from the $\sigma_{p}$-dual, then we are done; so suppose these duals coincide. We claim that in this case $\sigma_{p}$ is absolutely $p$-summing; so again $\sigma_{p} \neq \beta$, this time by Proposition 1 .

Recall that the norm on $E_{\beta}^{\prime}$ is the restriction of the $E^{\prime}$ norm. Suppose $\left(e_{n}\right)$ is a weakly $\sigma_{p}$ (hence $\beta$ ) p-summable sequence in $E$. Then, as in [7, $\S 1.2 .3$, p. 22], the set

$$
\left\{\sum_{1}^{N} a_{n} e_{n}: N=1,2, \cdots ; \sum\left|a_{n}\right|^{a} \leqq 1\right\},
$$

where $p^{-1}+q^{-1}=1$, is bounded in the weak topology induced on $E$ by $E_{\beta}^{\prime}$, hence strictly bounded by Mackey's theorem [5, §17.5, p. 155]. Since the strict and norm bounded subsets of $E$ coincide [1, Theorem 1], we have

$$
\sup \left|\sum_{1}^{N} a_{n}\left\langle e_{n}, e^{\prime}\right\rangle\right|<\infty,
$$

where the supremum is taken over all positive integers $N$, all sequences $\left(a_{n}\right)$ in the unit ball of $l^{q}$, and all $e^{\prime}$ in the unit ball of $E_{\beta}^{\prime}$. From this it follows easily that

$$
\sup \left\{\sum_{1}^{\infty}\left|\left\langle e_{n}, e^{\prime}\right\rangle\right|^{p}: e^{\prime} \in E_{\beta}^{\prime},\left\|e^{\prime}\right\| \leqq 1\right\}<\infty .
$$

Now if $S$ is a $\sigma_{p}$-continuous seminorm on $E$, then $S$ is bounded on a set of 
the form (3.2), hence $S \leqq S_{g}$ for some $g$ in $L^{+}$. Taking $\lambda_{s}$ as in (2.2) we obtain:

$$
\begin{aligned}
\sum\left(S e_{n}\right)^{\nu} & =\sum\left(S_{q} e_{n}\right)^{p}=\sum \int\left|e_{n}\right|^{p} g d \mu \\
& =\int\left(\sum\left|\left\langle e_{n}, \lambda_{s}\right\rangle\right|^{p}\right) g(s) d \mu(s) \leqq\|g\|_{1} \text { sup } \sum\left|\left\langle e_{n}, e^{\prime}\right\rangle\right|^{p}<\infty,
\end{aligned}
$$

where the supremum in the last line is taken over all $e^{\prime}$ in $E_{\beta}^{\prime}$ with $\left\|e^{\prime}\right\| \leqq 1$; a condition satisfied by each $\lambda_{s}$. That the supremum is finite follows from (3.3); hence $\sigma_{p}$ is an absolutely $p$-summing topology on $E$, and $\sigma_{p} \neq \beta$.

4. The bounded weak star and bounded strong operator topologies. Let $E$ be a subspace of $C(S)$, and let $b \sigma_{p}=b \sigma_{p}(\mu)$ denote the bounded strong operator topology induced on $E$ by its action on $L^{p}(\mu)$ (see [3, VI. 9.9, p. $512])$; that is, the strongest topology on $E$ agreeing with $\sigma_{p}$ on norm bounded sets.

If $X$ is a Banach space, then the bounded weak star topology on its dual $X^{\prime}$ is the strongest topology on $X^{\prime}$ agreeing with the weak star topology on bounded sets [3, V.3.3, p. 427]. According to the Banach-Dieudonné theorem [3, V.5.4], the bounded weak star topology on $X^{\prime}$ is just the topology of uniform convergence on null sequences of $X$. From this and Lemma 1 we get the following result, already noted by Lazar and Retheford for $X=c_{0}$ [6, Example 2, p. 417].

THEOREM 2. If $X$ is an infinite-dimensional Banach space, then the bounded weak star topology on $X^{\prime}$ is not absolutely p-summing. In particular, it is not nuclear.

In [10, Theorem 2, p. 475] we showed that if $E$ is a linear subspace of $C(S)$ whose unit ball is strictly compact, then $E$ is the dual of the quotient Banach space $M(S) / E^{\prime \prime}$, and the bounded weak star topology thus induced on $E$ is just the strict topology. This quickly yields the following

THEOREM 3. Suppose $E$ is a linear subspace of $C(S)$ whose unit ball is strictly compact. Let $\mu$ be a regular Borel measure on $S$. Then $b \sigma_{p}(\mu)=\beta$.

Proof. By [10, Theorem 2] $\beta$ is the strongest topology on $E$ agreeing on bounded sets with the weak topology induced by $M(S) / E^{\circ}=E_{\beta}^{\prime}$. The proof of Theorem 1 shows that $\sigma_{p} \leqq \beta$, so the unit ball of $E$ is also $\sigma_{p^{-}}$ compact. But the topology $\sigma_{p}$ is Hausdorff, so $\sigma_{n}=\beta$ on the unit ball of $E$, hence on every bounded set (since they are both vector topologies). Thus $b \sigma_{p}=\beta$.

In particular note that if $E$ is $H^{\infty}(G)$ and $\mu$ is Lebesgue measure on $G$, 
then the hypotheses of Theorem 3 are satisfied. Thus if $G$ supports nonconstant bounded analytic functions, then $H^{\infty}(G)$ is infinite-dimensional; and the strict topology on it is the bounded strong operator topology, but not the strong operator topology.

\section{REFERENCES}

1. R. C. Buck, Bounded continuous functions on a locally compact space, Michigan Math. J. 5 (1958), 95-104. MR 21 \#4350.

2. J. B. Conway, The strict topology and compactness in the space of measures. II, Trans. Amer. Math. Soc. 126 (1967), 474-486. MR 34 \#6503.

3. N. Dunford and J. T. Schwartz, Linear operators. I: General theory, Pure and Appl. Math., vol. 7, Interscience, New York, 1958. MR 22 \#8302.

4. P. R. Halmos, Measure theory, Van Nostrand, Princeton, N.J., 1950. MR 11, 504.

5. J. Kelley, I. Namioka et al., Linear topological spaces, University Series in Higher Math., Van Nostrand, Princeton, N.J., 1963. MR 29 \#3851.

6. A. Lazar and J. Retheford, Nuclear spaces, Schauder bases, and Choquet simplexes, Pacific J. Math. 37 (1971), 409-419.

7. A. Pietsch, Nukleare lokalkonvexe Räume, Schriftenreihe der Institute für Mathematik bei der Deutschen Akademie der Wissenschaften zu Berlin. Reihe A, Reine Mathematik, Heft 1, Akademie-Verlag, Berlin, 1965. MR 31 \#6114.

8. - Absolut p-summierende Abbildungen in normierten Räumen, Studia Math. 28 (1966/67), 333-353. MR 35 \#7162.

9. L. A. Rubel and A. L. Shields, The space of bounded analytic functions on a region, Ann. Inst. Fourier (Grenoble) 16 (1966), fasc. 1, 235-277. MR 33 \#6440.

10. J. Shapiro, Weak topologies on subspaces of $C(S)$, Trans. Amer. Math. Soc. 157 (1971), 471-479.

Department of Mathematics, Michigan State University, East lansing, MiCHIGAN 48823 